\title{
The choice of a measure of comparison in granular learning methods
}

\author{
Maria Rifqi \\ LIP6, mailbox 169 \\ Université P. et M. Curie \\ 4, place Jussieu \\ 75252 Paris cedex 05, France \\ Maria.Rifqi@lip6.fr
}

\author{
Vincent Berger \\ Laboratoire Central de Recherche \\ Thomson-CSF \\ Domaine de Corbeville \\ 91400 Orsay, France \\ berger@thomson-lcr.fr
}

\author{
Bernadette Bouchon-Meunier \\ LIP6, mailbox 169 \\ Université P. et M. Curie \\ 4, place Jussieu \\ 75252 Paris cedex 05, France \\ Bernadette.Bouchon-Meunier@lip6.fr
}

\section{Introduction}

The comparison of two objects is a usual task for many classification and learning methods.

Comparisons are usually evaluated through a measure of comparison. The used measure is often a distance. But, more and more, a similarity or a dissimilarity measure is chosen. But the choice of an appropriate measure among all avalaible measures in literature is not an easy task. It is linked to the problem of the characterization of relevant properties for the considered task. We have proposed [2] a framework in order to deal with measures of comparison. This framework displays the main families of measures of comparison according to the properties they satisfy. Hence, the existing measures of comparison can be classified. Fyrther, it is known that a classification simplifies a problem.

However, the problem of the choice of a measure of comparison within a same family is still present. This paper gives an explicit method to choose a measure of comparison.

The proposed solution lies in a new representation of measures of comparison obtained by normalization of arguments. This normalization leads to a desirable property: measures of comparison do not depend on the scale of the system. Another consequence of this normalization is the fact that measures of satisfiability can be described by a unique argument and measures of resemblance by two arguments. The analysis of behaviours of measures of comparison is easy thanks to a geometrical interpretation and to a natural definition of the power of discrimination of a given measure of comparison.

We focus on two families of measures of similitude: measures of satisfiability and measures of resemblance. For each family, we discuss the role of the new parameter we point out and we propose new measures satisfying particular properties. For measures of satisfiability, the parameter $\phi$ controls the severity towards difference between sets whereas the parameter $\rho$ penalizes the differences between two sets for a measure of resemblance.

\section{Measures of comparison}

In [2], we have proposed to formalize a measure of comparison between two fuzzy sets as a function of the common elements and the distinctive elements.

Formally, for any set $\Omega$ of elements, let $F(\Omega)$ denote the set of fuzzy subsets of $\Omega$.

Definition $1 A$ fuzzy set measure $M$ is supposed to be given, that is to say a mapping defined on $F(\Omega)$ and taking values in $\mathbb{R}^{+}$such that, for all $A$ and for all $B$ in $F(\Omega)$ :

MI1 : $M(A)=0 \Leftrightarrow A=\emptyset$.

MI2 : if $B \subseteq A$, then $M(B) \leq M(A)$.

where $\subseteq$ denotes the classical inclusion of fuzzy sets.

A fuzzy set measure is close to the definition of an existential evaluator given by [6].

Definition 2 An $M$-measure of comparison on $\Omega$ is a mapping $S: F(\Omega) \times F(\Omega) \rightarrow[0,1]$ such that $S(A, B)=$ $F_{S}(M(A \cap B), M(B-A), M(A-B))$, for a given mapping $F_{S}: \mathbb{R}^{+} \times \mathbb{R}^{+} \times \mathbb{R}^{+} \rightarrow[0,1]$ and a fuzzy set measure $M$ on $F(\Omega)$.

We denote:

- $X=M(A \cap B)$

- $Y=M(B-A)$

- $Z=M(A-B)$

where $\cap$ denotes the classical intersection of fuzzy sets and - is a difference of fuzzy sets defined by:

D1 : if $A \subseteq B$, then $A-B=\emptyset$. 
D2 : $B-A$ is monotonous with regard to $B: B \subseteq B^{\prime}$ entails $B-A \subset B^{\prime}-A$

We are interested in measures of comparison which evaluate the likeliness of two descriptions. We have called them measures of similitude.

Definition 3 An $M$-measure of similitude $S$ on $\Omega$ is an $M$ measure of comparison $S$ such that $F_{S}(X, Y, Z)$ is non decreasing with respect to $X$, non increasing with respect to $Y$ and $Z$.

$M$-measures of similitude can be distinguished more subtly in three types: measures of satisfiability, measures of resemblance and measures of inclusion. In this paper, we focus on two types of measures of similitude.

\subsection{Measures of satisfiability}

A measure of satisfiability corresponds to a situation in which we consider a reference object or a class and we need to decide if a new object is compatible with it or satisfies it. More particularly, measures of satisfiability are appropriate for rule base systems. For example, in [1] objects are classified by means of a decision tree. In [1], the comparison between the value of an attribute of the new example with test-values associated with each edge is realized by means of a measure of satisfiability.

The satisfiability of a reference description $A$ of $F(\Omega)$ by a new description $B$ defined as a fuzzy subset of $\Omega$ has been defined as follows:

Definition 4 An $M$-measure of satisfiability on $\Omega$ is a measure $S(A, B)=F_{S}(X, Y, Z)$ such that:

- $F_{S}$ is increasing with respect to $X$, decreasing with respect to $Y$ and independent of $Z$. Let us denote $F_{S} .(X, Y)=F_{S}(X, Y, Z)$. Then,

- $F_{S} \cdot(X, 0)=1$ for all $X$

- $F_{S} \cdot(0, Y)=0$ for all $Y \neq 0$

With this definition, the value of the satisfiability of $B$ for $A$ can a priori be different with two pairs of fuzzy sets distinctive only because of the scale. It is desirable that a satisfiability measure depends only on the relative weights of $X$ and $Y$ and not on the scale of the system. In order to obtain an objective measure, we propose to normalize the satisfiability measure.

We consider:

$$
\begin{aligned}
& x=\frac{X}{\sqrt{X^{2}+Y^{2}}} \text { the reduced intersection } \\
& y=\frac{Y}{\sqrt{X^{2}+Y^{2}}} \text { the reduced distinctive feature }
\end{aligned}
$$

As $x^{2}+y^{2}=1$, the domain of definition of the measure of satisfiability is a quarter of circle. It can be described by a unique argument $\phi$, with $\phi=\arctan \frac{y}{x}$. We denote the measure of satisfiability $S(A, B)=\eta(\phi)$.

The conditions of definition 4 become:

- $\eta$ is decreasing with respect to $\phi$.

- $\eta\left(\frac{\pi}{2}\right)=0$

- $\eta(0)=1$

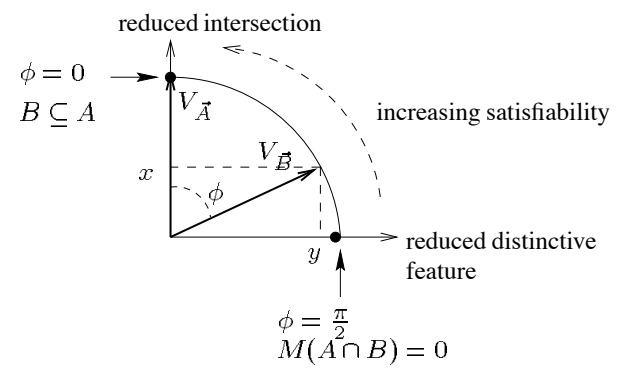

Figure 1. New representation of a measure of satisfiability

This new form of a measure of satisfiablity, expressed by a unique variable, has the advantage not to be dependent upon the size of the system. Furthermore, this normalization makes the definition of a measure of satisfiability more simple insofar as the argument is a segment $\left[0, \frac{\pi}{2}\right]$ and not a quarter of plan.

There are of course many possible choices for the satisfiability measure $\eta$ satisfying these three conditions. Among them, let us distinguish the two following forms:

- $\eta_{1}(\phi)=1-\frac{2}{\pi} \phi$. It is a linear satisfiability function.

- $\eta_{2}(\phi)=\cos \phi$. This function has the advantage of presenting a meaningful physical insight. If we represent the reference set $A$ by the $(1,0)$ vector $V_{\vec{A}}$ in figure 1 . If we describe each set $B$ and its related point $(x, y)$ by a vector $V_{\vec{B}}$ from the origin, $\eta_{2}(\phi)$ is the scalar product $V_{\vec{A}} \cdot V_{\vec{B}}$. When the two vectors are orthogonal, then the satisfiability vanishes: $S(A, B)=0$. This is a good signification of orthogonality. More generally, the satisfiability appears as a projection, and the lack of satisfiability is represented as a deviation in figure 1 : this is an intuitive notion of satisfiability.

We can also focus on the following measures which are known in the literature:

- $S(A, B)=\frac{M(A \cap B)}{M(B)}$, which is usually defined with $M$ the sigma-count, can be also written: $\eta_{3}(\phi)=\frac{1}{1+\tan \phi}$. 
- $S(A, B)=1-M(B-A)$ was introduced in [3] with $M(A)=\sup _{x} f_{A}(x)$ and with the difference $f_{A-B}(x)=\left\{\begin{array}{l}f_{A}(x) \text { if } f_{B}(x)=0 \\ 0 \text { if } f_{B}(x)>0\end{array}\right.$. It can be also written: $\eta_{4}(\phi)=1-\sin \phi$.

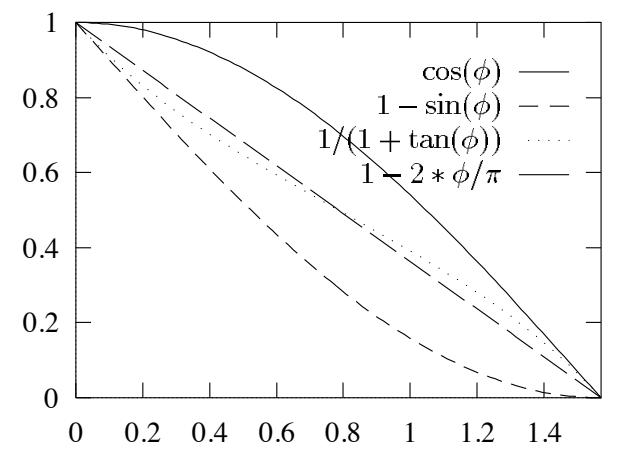

Figure 2. Satisfiability measures

Figure 2 displays the behaviour of various mentioned measures of satisfiability. We can see that:

- $\eta_{1}$ is linearly discriminant: satisfiability decreases linearly with the deviation.

- $\eta_{2}$ is discriminant for low satisfiability: a small difference between a set and the reference is tolerated.

- $\eta_{4}$ is discriminant for high satisfiablity: a small difference between a set and the reference is not tolerated.

- $\eta_{3}$ is discriminant for high and low satisfiability at the same time. For high satisfiability, this measure is between the linearly discriminant measure and the low satisfiability discriminant measure. For low satisfiability, it is between the linearly discriminant measure and the high satisfiability discriminant measure. But it is low discriminant for $\eta(\phi)=1 / 2$.

Remark $\eta_{4}$ and $\eta_{2}$ are symmetrical relatively to $\left(\frac{\pi}{4}, \frac{1}{2}\right)$ : $\eta_{4}\left(\frac{\pi}{2}-\phi\right)=1-\eta_{2}(\phi) . \eta_{2}$ has a low discrimination power for high satisfiability, and a high discrimination power for poor satisfiability, whereas $\eta_{4}$ does exactly the reverse.

We can consider that the discrimination power of a measure of satisfiability is given by the derivative $\eta^{\prime}(\phi)$ of $\eta$. For instance, for small and large differences between a set and the reference that is to say for $\phi=0$ or $\phi=\frac{\pi}{2}$ :

- $\eta_{1}^{\prime}(\phi)=-\frac{2}{\pi}$, for all $\phi$.

- $\eta_{2}^{\prime}(0)=0$ and $\eta_{2}^{\prime}\left(\frac{\pi}{2}\right)=-1$

- $\eta_{3}^{\prime}(0)=-1$ and $\eta_{3}^{\prime}\left(\frac{\pi}{2}\right)=-1$

- $\eta_{4}^{\prime}(0)=-1$ and $\eta_{4}^{\prime}\left(\frac{\pi}{2}\right)=0$
In general, for every possible $\eta$, we have:

$$
\int_{0}^{\frac{\pi}{2}} \eta^{\prime}(\phi) d \phi=\eta\left(\frac{\pi}{2}\right)-\eta(0)=-1
$$

This means that the total discrimination power $\eta^{\prime}(\phi)$ has to be distributed on the $\left[0, \frac{\pi}{2}\right]$ interval, but a high discrimination power somewhere implies a low discrimination power elsewhere, the integral being constant.

Accordingly, it is necessary to choose a measure with a discrimination power suitable for the considered application. This suggests a method of construction of a measure of satisfiability. The choice of the discrimination power is the first step. Then $\eta$ is obtained by integration of this function $\eta^{\prime}(\phi)$.

For instance, a function with a high discrimination power for $\eta(\phi)=1 / 2$ but a low discrimination for $\eta(\phi)=0$ and $\eta(\phi)=1$ is needed. This kind of measures means that if a description is not far from the reference, then the satisfiability is near from 1 because the difference is not significative. If a description is very far from the reference, we can consider that the satisfiability is null.

Once the behaviour of the wanted measure is known, the measure $\eta$ can be computed thanks to the discrimination power $\eta^{\prime}$.

We would like to give the example of an interesting function for the measure of satisfiability using the Fermi-Dirac function. The analytic form is:

$$
F_{F D}(\phi)=\frac{1}{1+\exp \frac{\left(\phi-\frac{\pi}{4}\right)}{\Gamma}}
$$

and

$$
\eta(\phi)=\frac{F_{F D}(\phi)-F_{F D}\left(\frac{\pi}{2}\right)}{F_{F D}(0)-F_{F D}\left(\frac{\pi}{2}\right)}
$$

$\eta(\phi)$ reflects essentially the Fermi-Dirac function. The above expression is used to ensure that $\eta(0)=1$ and $\eta\left(\frac{\pi}{2}\right)=0$.

The interest of this function lies on its physical meaning: in a physical system of temperature $T$, containing a statistical set of states, the Fermi-Dirac function describes the statistical probability that a state of energy $\phi$ is filled or not, with $\Gamma=k T$, where $k$ is the Boltzmann constant. Hence, it describes the probability of a state to belong to the Fermi sea [4] which is a good illustration of satisfiability. $\Gamma$ controls the decrease of the curve. The choice of $\Gamma$ enables to define a measure of satisfiability more or less severe, as shown on figure 3 .

\subsection{Measures of resemblance}

We are now interested in resemblance measures.

A measure of resemblance is used for a comparison between the descriptions of two objects, of the same level of 


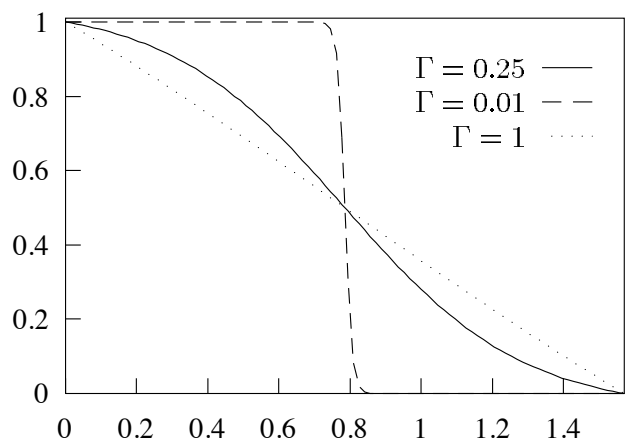

Figure 3. The Fermi-Dirac measure of satisfiability

generality, to decide if they have many common characteristics.

Measures of resemblance are appropriate for a casebased reasoning or an instance-based learning. In clustering methods, distances can be replaced by a measure of resemblance. More generally, similarity-based classification methods [7], [8] have to use resemblance measures as soon as all objects have the same level of generality.

Definition 5 An $M$-measure of resemblance on $\Omega$ is a measure $S(A, B)=F_{S}(X, Y, Z)$ such that:

- $F_{S}$ is increasing with $X$ and decreasing with $Y$ and $Z$

- $F_{S}(X, 0,0)=1$ for all $X$

- $F_{S}(X, Y, Z)=F_{S}(X, Z, Y)$.

$M$-measures of resemblance which satisfy an additional property of $t$-transitivity, for a triangular norm $t$, are extensions of indistinguishability relations [9], [10] to fuzzy sets. In the case where $t$ is the minimum, we obtain extensions of measures of similarity.

$M$-measures of resemblance satisfying the property of exclusiveness:

$$
F_{S}(0, Y, Z)=0 \text { for all }(Y, Z) \neq(0,0)
$$

are called exclusive $M$-measures of resemblance. We focus on them in the sequel.

Following our normalization procedure, we define:

$$
\begin{aligned}
& x=\frac{X}{\sqrt{X^{2}+Y^{2}+Z^{2}}} \\
& y=\frac{Y}{\sqrt{X^{2}+Y^{2}+Z^{2}}} \\
& z=\frac{Z}{\sqrt{X^{2}+Y^{2}+Z^{2}}}
\end{aligned}
$$

for $(X, Y, Z) \neq(0,0,0)$. Similarly to the case of measures of satisfiability, this ensures that an exclusive measure of resemblance is not dependent on the scale of the problem.

The domain of study is now restricted to a piece of the unity sphere since $x^{2}+y^{2}+z^{2}=1$. We have now to find the exclusive resemblance $\mu$ which satisfies the symmetry property $\mu(x, y, z)=\mu(x, z, y)$.

Geometrically, the sphere is simply obtained by a rotation of the satisfiability circle around the $x$-axis (see figure 4). The vector representation is still valid.

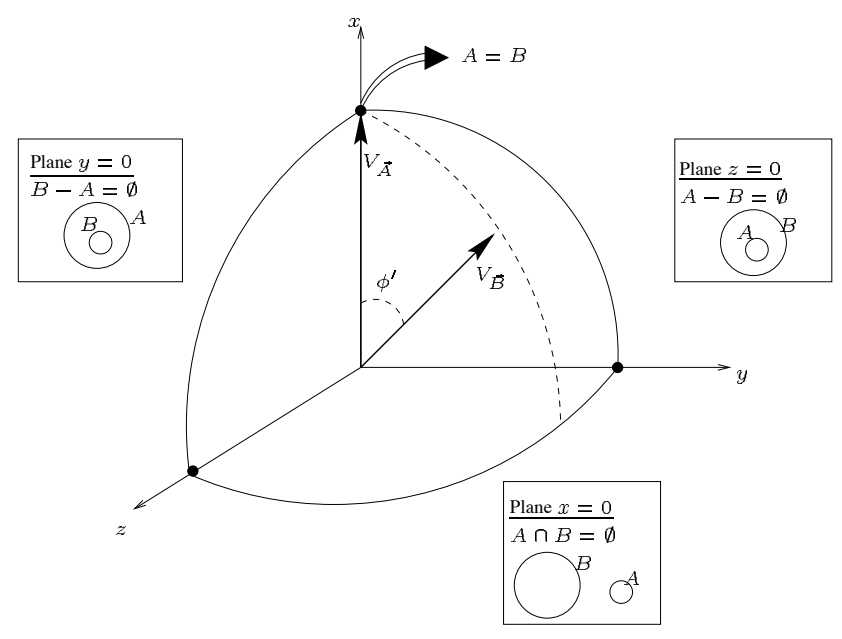

Figure 4. New representation of an exclusive measure of resemblance

By definition, an exclusive measure of resemblance is symmetrical with respect to $(Y, Z)$, (see Definition 5). Let us consider $\rho=\xi(y, z)$ with $\xi(y, z)=\xi(z, y)$. This means that $\rho$ can be described by any monotonous symmetrical function with respect to $y$ and $z$ and such that $\xi(0,0)=0$.

Let us look for an expression of $\nu(x, \rho)=\mu(x, y, z)$. We have:

$$
\begin{aligned}
& \nu(0, \rho)=0 \text { if } \rho \neq 0 \\
& \nu(x, 0)=1 \forall x
\end{aligned}
$$

These conditions show that the problem has been reduced to a satisfiability measure. We can therefore use again the solution described in the preceding section dealing with satisfiability. With this definition of $\rho$, an exclusive resemblance appears as a satisfiability where a global distinctive feature $\rho$ is defined by $\rho=\xi(y, z)$, from the two individual distinctive features $y$ and $z$ (see Figure 5).

We can also consider different exclusive measures of resemblance as we have already done with measures of satis- 


Satisfiability
$(x, y) \rightarrow$ with $\rho=\xi(y, z)$

Figure 5. Correspondance between satisfiability measures and exclusive resemblance measures

fiability. Let us define $\psi=\arctan \left(\frac{\rho^{0}}{x}\right)$ with $\rho^{0}=y+z$.

- $\nu_{1}=\frac{1}{1+\frac{\rho^{\omega}}{\alpha}}=\frac{1}{1+\tan \psi}$. This measure corresponds to the measure of satisfiability $\eta_{3}$. Furthermore, $\nu_{1}$ can be also written as: $S(A, B)=\frac{M(A \cap B)}{M(A \cup B)}$ with $M$ such that: $M(A \cup B)=M(A \cap B)+M(A-B)+M(B-A)$. This measure was introduced in [5].

Other definitions of $\rho$ can be envisaged, for instance:

$$
\begin{aligned}
\rho^{\prime} & =\sqrt{y^{2}+z^{2}} \\
\text { or } \rho^{\prime \prime} & =(\sqrt{y}+\sqrt{z})^{2}
\end{aligned}
$$

The choice of a particular form of $\rho$ has an effect on the measure of resemblance because this parameter represents distinctive elements. We can notice that:

$$
\rho^{\prime \prime} \geq \rho^{0} \geq \rho^{\prime} \forall y, z
$$

As $\rho$ has a decreasing effect on an exclusive measure of resemblance, relation (1) implies that, for a given $x$ and for all $y$ and $z$,

$$
\nu^{\prime \prime}\left(x, \rho^{\prime \prime}\right) \leq \nu\left(x, \rho^{0}\right) \leq \nu^{\prime}\left(x, \rho^{\prime}\right)
$$

Relation (2) means that $\nu^{\prime \prime}\left(x, \rho^{\prime \prime}\right)$ penalizes more the differences between two sets than $\nu\left(x, \rho^{0}\right)$ and that $\nu\left(x, \rho^{0}\right)$ penalizes more the differences than $\nu^{\prime}\left(x, \rho^{\prime}\right)$. Furthermore, a particular $\rho$ is sensitive to the symmetry between $y$ and $z$. Indeed, if differences are unbalanced, it means that $y>>z$ or $z \gg>y$, or inversely, if differences are balanced, it means that $y \approx z$, the behaviours of a given $\rho$ are not the same.

\section{Conclusion}

This paper enables to better analyse the behaviour of a measure of similitude thanks its discrimination power. This analysis is very useful in learning methods in general because a lot of them are based on comparisons of attribute values of objects in the learning database. Furthermore, the classification step is also often based on comparisons.

Each time a method needs a similitude measure, the questions are : when has the measure to be severe ? when has it to be tolerant ? The answers are given by the discrimination power described in this paper. We have given an example of a measure of satisfiability, the Fermi-Dirac function, with a parameter that controls the severity towards the difference with respect to the reference.

\section{References}

[1] B. Bouchon-Meunier, C. Marsala, and M. Ramdani. Learning from imperfect data. In H. P. D. Dubois and R. R. Yager, editors, Fuzzy Information Engineering: a Guided Tour of Applications, pages 139-148. John Wileys and Sons, 1997.

[2] B. Bouchon-Meunier, M. Rifqi, and S. Bothorel. Towards general measures of comparison of objects. Fuzzy Sets and Systems, 84(2):143-153, 1996.

[3] B. Bouchon-Meunier and L. Valverde. Analogy relations and inference. In Proceedings of $2^{\text {nd }}$ IEEE International Conference on Fuzzy Systems, pages 11401144, San Fransisco, 1993.

[4] B. P. Course. Statistical physics. vol. 5.

[5] D. Dubois and H. Prade. Fuzzy Sets and Systems, Theory and Applications. Academic Press, New- York, 1980 .

[6] D. Dubois and H. Prade. A unifying view of comparison indices in a fuzzy set-theoretic framework. In R. R. Yager, editor, Fuzzy set and possibility theory, pages 3-13. Pergamon Press, 1982.

[7] M. Rifqi. Constructing prototypes from large databases. In IPMU'96, pages 301-306, Granada, 1996.

[8] M. Rifqi, S. Bothorel, B. Bouchon-Meunier, and S. Muller. Similarity and prototype based approach for classification of microcalcifications. In $7^{\text {th }}$ IFSA World Congress, pages 123-128, Prague, 1997.

[9] E. Trillas and L. Valverde. On implication and indistinguishability in the setting of fuzzy logic. In J. Kacprzyk and R. R. Yager, editors, Management Decision Support Systems Using Fuzzy Sets and Possibility Theory. Verlag TUV, Rheinland, 1984.

[10] L. Valverde. On the structure of t-indistinguishability operators. Fuzzy Sets and Systems, 17:313-328, 1985. 